\title{
Aportes científicos a la gestión empresarial desde Latinoamérica
}

\author{
Scientific contributions to business management from Latin America
}

Contribuições científicas para a gestão de negócios da América Latina

Recibido: octubre 2019

Arbitrado: noviembre 2019

Aceptado: diciembre 2019

Publicado: enero 2021 $\triangle$ Laura Raquel Cardozo Rejas

xlauracardozorejas@gmail.com

https://orcid.org/0000-0003-2496-9847

Universidad Privada de Valle, Santa Cruz-Bolivia

\section{RESUMEN}

El objetivo de este artículo fue analizar la participación de Latinoamérica en la difusión de conocimientos científico de alto impacto en áreas de negocios, administración y contabilidad. Con un enfoque cuantitativo $y$ corte longitudinal se censaron dos poblaciones: 1448 revistas científicas de la categoría "negocios, administración y contabilidad" indizadas en la base de datos de Scopus y 184 países con al menos una publicación indizada a esta base de datos bibliográfica. Las variables consideradas fueron: procedencia de revistas y país de afiliación; periodos de publicación, referencias y citas; tipo de acceso; indizaciones en Scopus, SciELO y WoS; índices $\mathrm{h}$, JCR, SJR. Se obtuvo como resultado, la cantidad de publicaciones por revistas latinoamericanas es inferior a la cantidad de publicaciones con afiliación en la región. Durante el estudio la producción científica presentó crecimiento constante, se deberá dar un salto exponencial para lograr una participación considerable a nivel mundial.

Palabras clave: Bibliometría; producción científica; informetría; latinoamérica; administración, contabilidad
The objective of this article was to analyze the participation of Latin America in the dissemination of high impact scientific knowledge in the areas of business, administration and accounting. With a quantitative approach and longitudinal cut, two populations were census: 1448 scientific journals in the category "business, administration and accounting" indexed in the Scopus database and 184 countries with at least one publication indexed to this bibliographic database. The variables considered were: origin of journals and country of affiliation; periods of publication, references and citations; type of access; indexes in Scopus, Scielo and Wos; h, JCR, SJR indices. As a result, the number of publications by Latin American magazines is lower than the number of publications with affiliation in the region. During the study, the scientific production presented constant growth, an exponential leap must be taken to achieve a considerable participation worldwide.

Key words: Bibliometrics; scientific production; Reporting; latinoamerica; management accounting

\section{RESUMO}

O objetivo deste artigo foi analisar a participação da América Latina na difusão do conhecimento científico de alto impacto nas áreas de negócios, administração e contabilidade. Com abordagem quantitativa e corte longitudinal, duas populações foram censuradas: 1448 periódicoscientíficos na categoria "negócios, administração e contabilidade" indexados na base de dados Scopus e 184 países com pelo menos uma publicação indexada nesta base bibliográfica. As variáveis consideradas foram: origem dos periódicos e país de afiliação; períodos de publicação, referências e citações; tipo de acesso; índices em Scopus, SciELO e WoS; índices h, JCR, SJR. Como resultado, o número de publicações de revistas latinoamericanas é inferior ao número de publicações afiliadas na região. Durante o estudo, a produção científica apresentou crescimento constante, um salto exponencial deve ser dado para alcançar uma participação considerável em todo o mundo.

Palavras chave: Bibliometria; produção científica; Comunicando; América latina; contabilidade gerencial 


\section{INTRODUCCIÓN}

$\mathrm{S}$ egún el análisis presentado por Ortiz y Viamonte (2020), la investigación sobre el liderazgo científico en el contexto iberoamericano es insuficiente; si bien ésta presenta puntos de contacto comunes al resto de las categorías de liderazgo altamente estudiadas (político, educativo y comunitario), existen particularidades lógicas y dinámicas propias de su ethos que demandan independencia en su abordaje. En este sentido, Ortiz y Viamonte (2020), SCImago Research Group (2014), Urbizagastegui (2016), Díaz y Vitón (2020), se han interesado por identificar y analizar la participación de autores de impacto en la generación de conocimiento.

Asimismo, otros autores han estudiado la difusión del conocimiento desde la perspectiva de las revistas científicas: Spinak (1998), Delgado et al (2006), Cañedo y Dorta (2010); Benet y Morejón (2016), desde la perspectiva del área de salud en Cuba al 2016. Domínguez et al. (2018); Romaní y Cabezas (2018), desde la perspectiva del área de salud en Perú tomando datos del periodo 2010-2017; Velásquez y Tocuyo (2021).

Para publicaciones periódicas, los índices de impacto son considerados indicadores de calidad. Estos se clasifican en función de: revista, artículos, productividad y producción. Los indicadores más relevantes son: factor de impacto de las revistas, factor de impacto de los artículos, factor de impacto ponderado, factor de impacto acumulado, factor de impacto del autor, número de artículos con factor de impacto, índice de inmediatez, índice h, índice de internacionalidad, índice de citas acumuladas, índice de citas por artículo e índice de citas por año (Buela, 2010). Asimismo, Torres y Jiménez (2010), mencionan: el Journal Citation Reports incluyó los índices Eigen factor y Article influence score; además, Elsevier incluyó en Scopus los indicadores Source Normalized Impact per Paper (SNIP) y SCImago Journal Rank (SJR). Por su parte, Cabezas y Torres (2010), sugieren otros indicadores de escasa participación en rankings: número de comentarios, puntuación, número de bookmarks, enlaces desde blogs científicos y número de descargas. Oliván y Lacruz (2015), presentan un estudio correlacional de indicadores bibliométricos considerando publicaciones en WoS y Scopus concluyendo que algunos de estos indicadores son innecesarios por ser repetitivos; Torres y Jiménez (2010) coinciden y afirman que existe redundancia.

Las principales plataformas de evaluación de revistas científicas vigentes son; Scopus, Elsevier Journal Finder, Elsevier Journal Insights, Redalyc y Google Scholar Metrics, todas ellas proporcionan indicadores que evalúan las publicaciones académicas contenidas en sus propios repositorios: Coincidencia, del gestor bibliográfico EndNote, permite comparar el factor de impacto, categoría y cuartil de la revista en Journal of Citation Report; SCImago Journal \& Country Rank, evalúa las revistas científicas contenidas en la base de datos Scopus. La Matriz de Información para el Análisis de Revistas (MIAR), identifica las publicaciones por su ISSN y analiza los factores de impacto. Por su parte, Publish or Perish (POP) y Scholarometer, son aplicaciones que permiten analizar métricas en publicaciones periódicas. Altmetrics, son indicadores de actividad académica en redes sociales. 
SCImago Journal \& Country Rank provee indicadores cienciométricos e informétricos que permiten seguir el comportamiento y el impacto de contribuciones científicas a escala internacional; emplea la colección de literatura disponible en Scopus. A través de su portal se puede clasificar las publicaciones procesadas por SciELO y WoS; haciendo a su vez una clasificación entre publicaciones de acceso abierto y de acceso mediante suscripción.

Diversos estudios se han beneficiado de esta plataforma para medir los alcances de la divulgación científica: Grupo Scimago (2008), analizó la producción científica en Asia y determinó el impacto de éstas según el número de citas obtenidas; Zacca et al (2014), analizan el impacto de las publicaciones de Latinoamérica con énfasis en el área de "Salud pública"; Santesteban (2013), caracterizó el desempeño científico de Argentina entre 2001 y 2010, en las categorías "Ciencias agrícolas y biológicas, Bioquímica, Genética y Biología molecular, Energía, Ciencia de los materiales, Física y Astronomía"; Gómez et al. (2016), desarrollaron un cienciograma basado en citas populares de las revistas Scopus, útil para evaluar clasificaciones, recuperar información y analizar dominios; Baena y Alzate (2020), describen la categoría "Negocios internacionales" según tipo de publicación (artículos, libros e informes de conferencias) y país de procedencia.

Los estudios antes citados aportan desde diferentes perspectivas a la evaluación de liderazgo científico de los diferentes países de Latinoamérica en particular y del mundo en general. Sin embargo, se advierte que aún existen bases de datos confiables de acceso abierto que, bajo un tratamiento adecuado, podrían proveer conocimiento oportuno respecto a la participación, evolución e impacto de los países latinos en la difusión del conocimiento. En este sentido, el objetivo de la investigación es analizar la participación de Latinoamérica en la difusión de conocimientos científico de alto impacto en áreas de negocios, administración y contabilidad.

\section{MÉTODO}

e presentó un estudio de corte longitudinal realizado bajo un enfoque cuantitativo que aplicó censos a las siguientes poblaciones: 1 ) revistas científicas de la categoría "negocios, administración y contabilidad" indizadas en la base de datos bibliográfica de Scopus hasta el año 2019; 2) países con al menos un investigador que haya publicado en una revista indizada en Scopus al año 2019. En este sentido, la investigación se desarrolló en dos etapas: primero, se ejecutó un análisis de la participación e impacto de revistas científicas según país de procedencia en un período que abarcó de 1999 a 2019; segundo, se estudió la clasificación de países según la participación e imparto generado por sus investigadores en un periodo de tiempo comprendido entre 1996 y 2019. En ambos casos la descarga de información se realizó en enero 2021 del sitio oficial de SCImago Journal \& Country Rank.

En la primera etapa, el universo censal estuvo conformado por 1448 revistas científicas procedentes de todos los países del mundo indizadas en Scopus, registradas en la categoría "negocios, administración y contabilidad" al 2019. Si bien no fue necesario aplicar criterios de exclusión, es importante resaltar que sólo se consideraron revistas científicas; quedando 
fuera del universo de interés: revistas de negocio, serie de libros, conferencias y congresos. El periodo de tiempo abarcó todos los años disponibles en esta base de datos a enero 2021 , es decir de 1999 a 2019. Los criterios de selección mencionados se muestran de manera gráfica en la Figura 1.

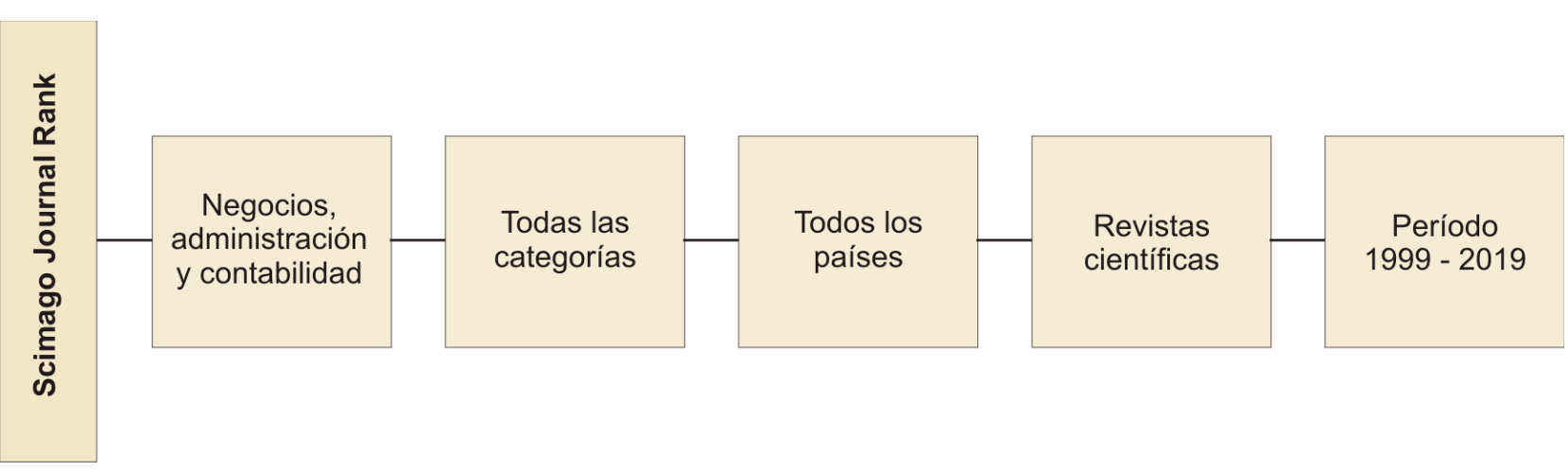

Figura 1. Criterios de selección de la información para la clasificación de revistas.

En la segunda etapa, el universo censal estuvo conformado por 184 países que cumplieron con el criterio de contar con al menos un investigador nacional que haya publicado en una revista registrada en la categoría "negocios, administración y contabilidad" de Scopus al 2019. Dadas las opciones habilitadas en la plataforma, se consideraron todos los artículos originales, de revisión y publicaciones en memorias de congresos. El período de tiempo abarcó todos los años disponibles a enero 2021, es decir de 1996 a 2019. Estos criterios de selección se presentan de manera gráfica en la Figura 2.

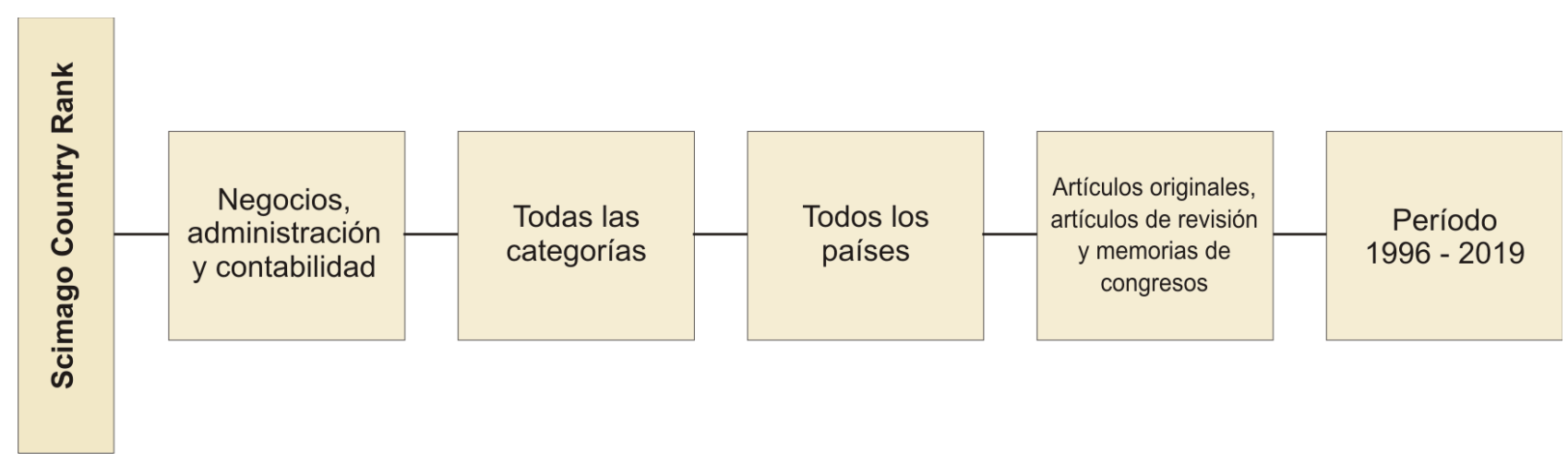

Figura 2. Criterios de selección de la información para la clasificación de países. 
El análisis en ambos casos, figuras 1 y 2, tuvo un alcance descriptivo. Las herramientas usadas fueron: Excel de Microsoft; portal SCImago Journal \& Country Rank, en las opciones disponibles para categorización de revista y países; y la aplicación de acceso abierto de lenguaje de secuencias de comandos Autohotkey.

\section{RESULTADOS}

on el propósito de analizar la participación de Latinoamérica en la difusión de conocimientos científico con impacto en áreas de negocios, administración y contabilidad en las últimas dos décadas, se determinó usar las funciones y base de datos disponibles en SCImago Journal \& Country Rank. Gracias a los criterios de selección de esta plataforma se garantizó que el análisis corresponda a revistas y artículos de alto impacto a nivel regional y mundial. Asimismo, se valoró contar con información exhaustiva de veintiún periodos anuales referente a: procedencia de la revistas y afiliación; periodos de publicación, referencias y citas; tipo de acceso; indizaciones en Scopus, SciELO y WoS; índices h, JCR, SJR; segmentación por subcategorías temáticas; y agrupación según región.

Los resultados se consolidaron en dos secciones: primero, se presentaron la caracterización de 1448 revistas científicas procedentes de todos los países del mundo indizadas en Scopus; segundo, se hizo una relación de 184 países que publicaron en al menos una revista registrada en la categoría "negocios, administración y contabilidad" de SCImago Journal \& Country Rank al 2019.

\section{Clasificación de revistas científicas a nivel mundial (1999-2019)}

El abordaje partió por observar el comportamiento en un periodo de veintiún años del total de revistas científicas indizadas en Scopus, considerando las siguientes regiones de origen: Asia, Europa Oriental, Norte América, Europa Occidental, África, África Medio Oriente, Latinoamérica, Oriente Medio y Región Pacífica (ver Figura 3).

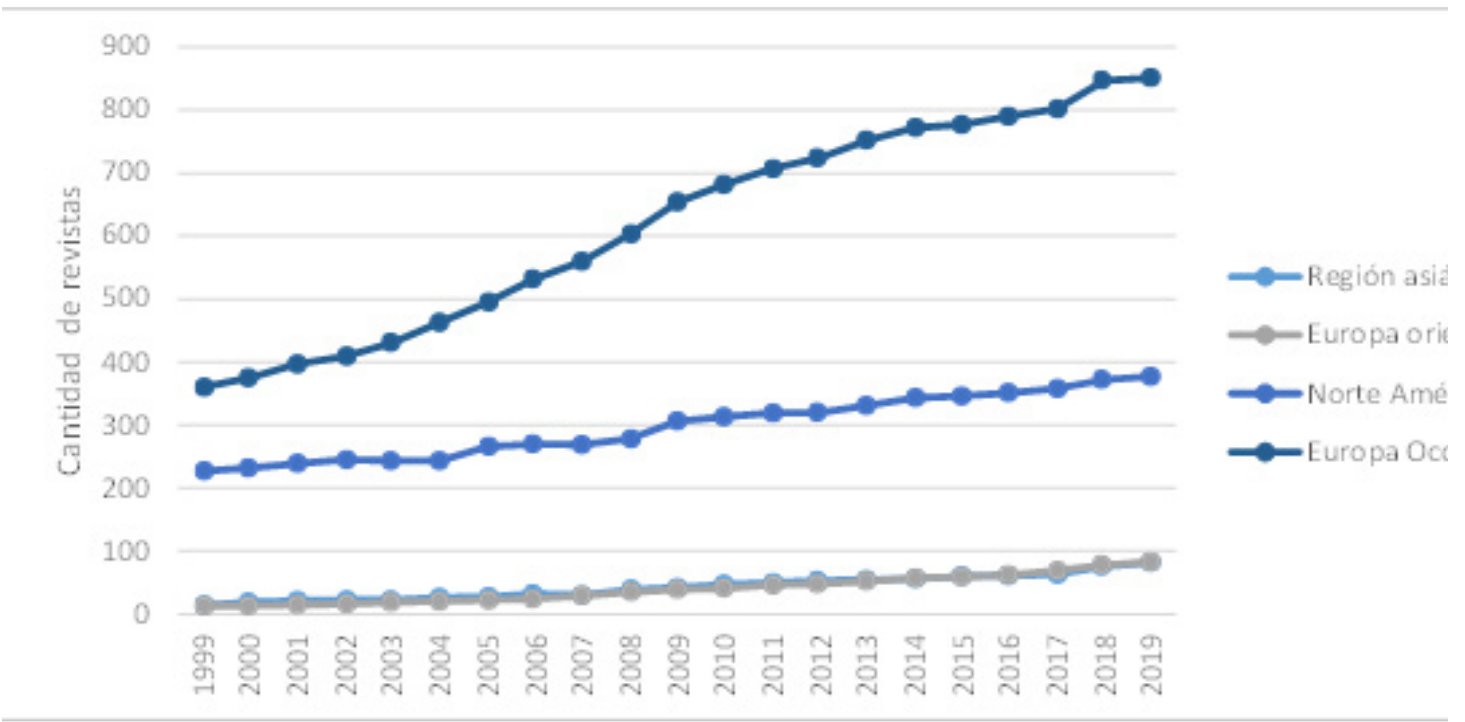




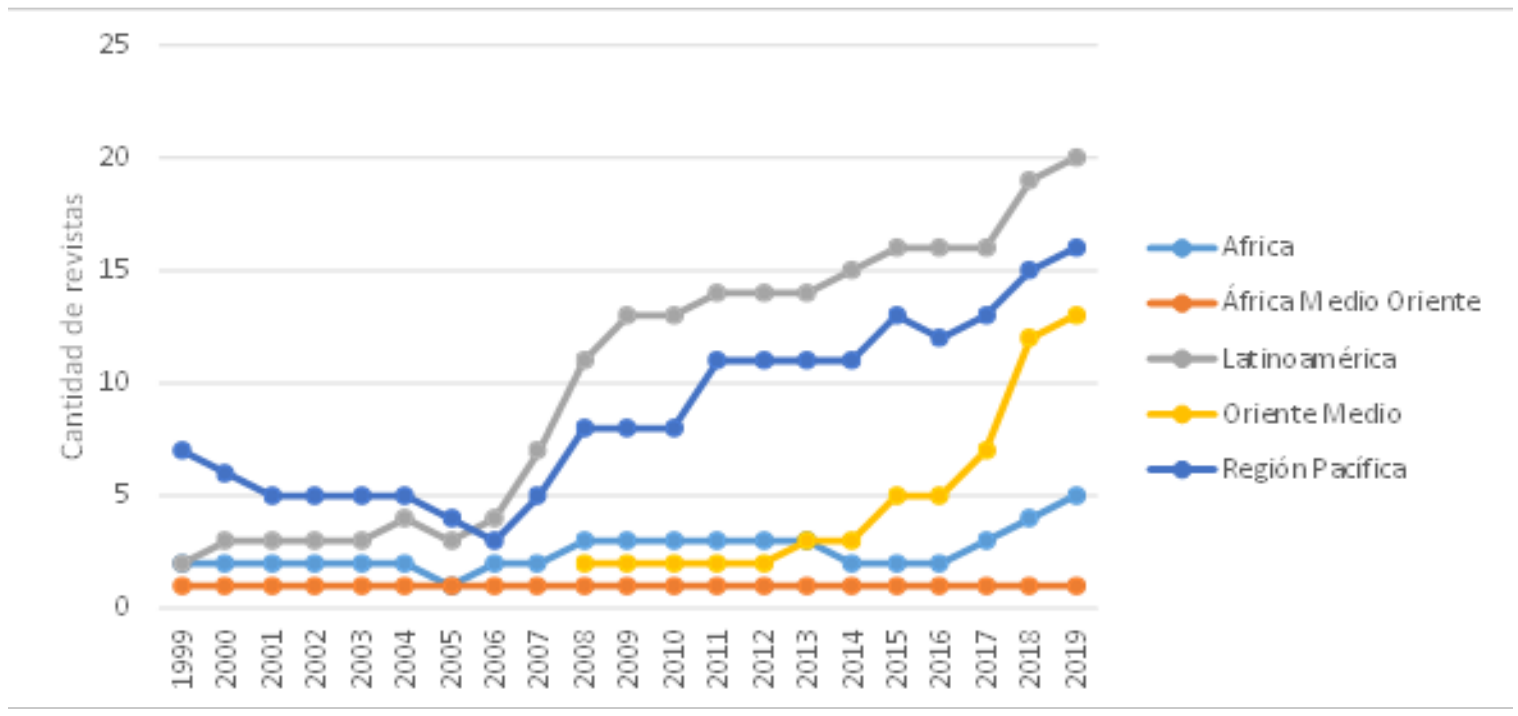

Figura 3. Cantidad de revistas científicas por región (1999-2019).

En la parte superior de la figura 3, se muestra que, Europa Occidental tiene el mayor número de revistas científicas dentro del universo poblacional estudiado; en los últimos veintiún años muestra una marcada tendencia a nuevos registros de revistas; en diecisiete años ha duplicado su participación. Europa oriental, Norte América y Asia, también han experimentado un incremento constante a través de los años; sin embargo, la pendiente es menor. El panorama es otro en Latinoamérica, Oriente Medio y Región Pacífica (parte inferior de la figura 3), si bien se observa una marcada tendencia al incremento de revistas científicas a partir del año 2006, las cantidades de revistas publicadas están lejos de la producción científica de Asia, Norte América y Europa. Como ejemplo, se mencionó, las revistas científicas de origen latinoamericano representan un $2 \%$ de las revistas de Europa Occidental. Finalmente, África Medio Oriente se ha mantenido por diecisiete años con tres revistas científicas indexadas en Scopus; a partir de 2017 demuestra una tendencia incremental. Este mismo análisis se amplía para Latinoamérica en la Figura 4. 
12

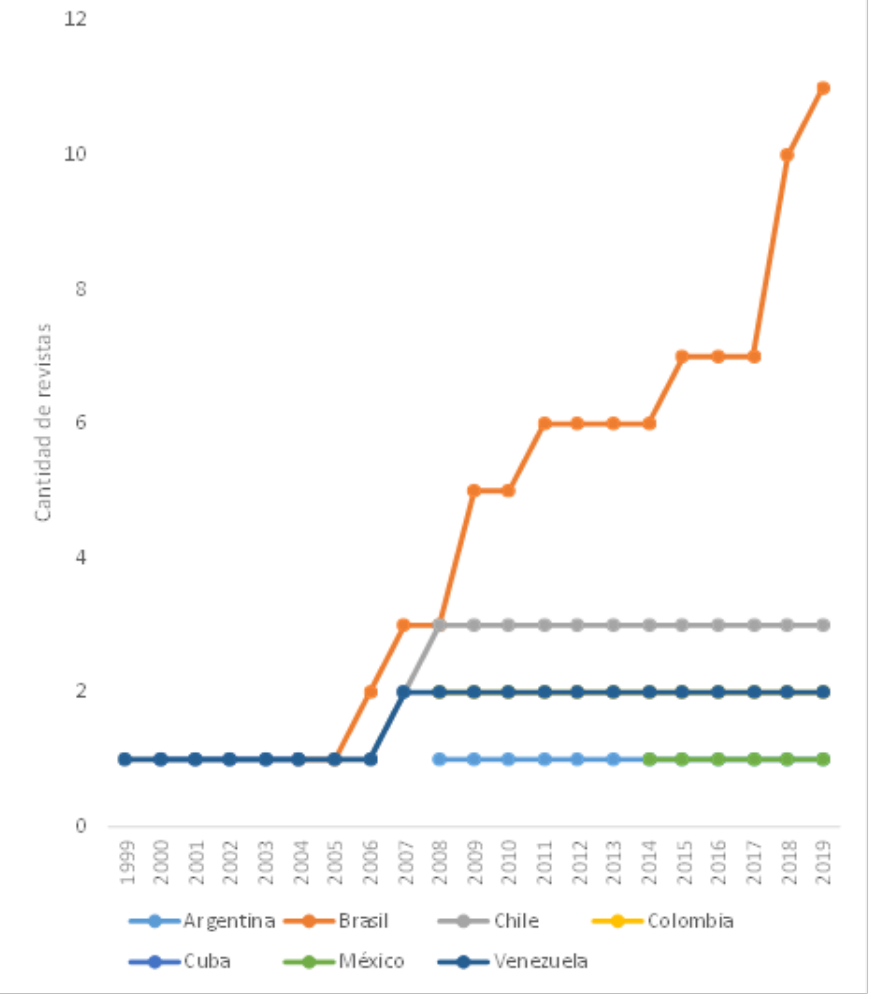

2500

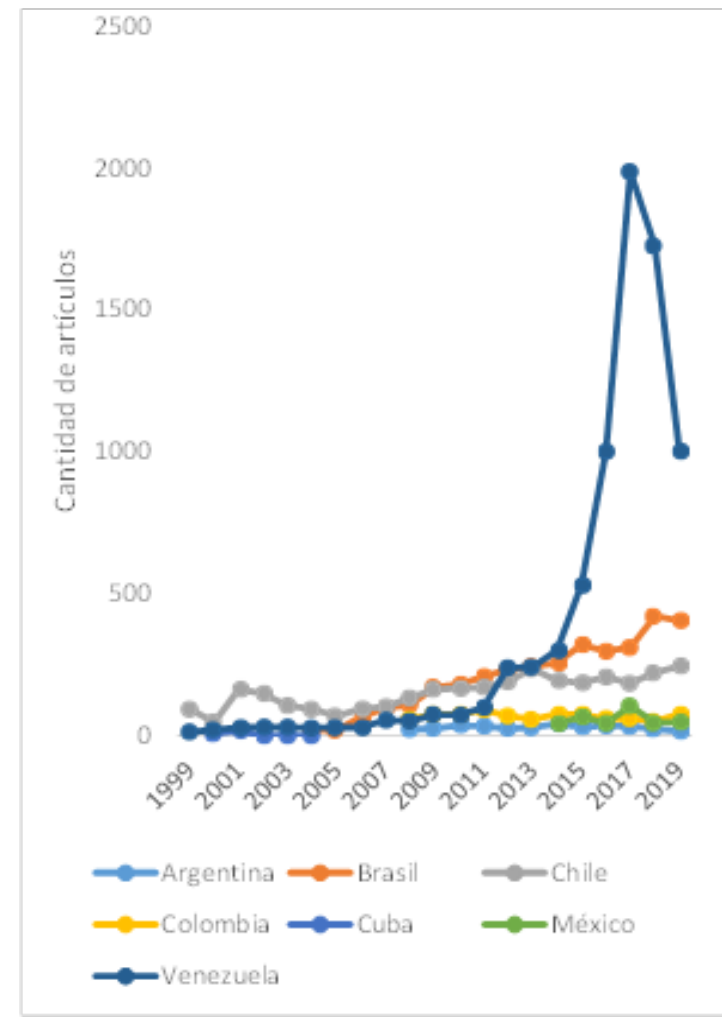

Figura 4. Revistas y artículos científicos de origen latinoamericano (1999-2019).

A la izquierda de la Figura 4 resalta Brasil, que tras un incremento constante que inició en 2005 llegó a tener 11 revistas científicas presentes en Scopus el 2019. Sin embargo, cuando se mide la cantidad de artículos publicados por las revistas (lado derecho), no es Brasil quien lleva la delantera; Venezuela con solo dos revistas científicas llegó a publicar 1987 artículos en 2017, de las que 1959 fueron publicadas por la Revista de acceso restringido Espacios; en los siguientes años se advierte una caída vertiginosa llegando en 2019 a tener 1002 publicaciones, 995 corresponden a Espacios, revista que a la actualidad ha sido descontinuada.

Respecto a los demás países de Latinoamérica con presencia es Scopus (Chile, México, Cuba, Colombia y Argentina), el número de revistas presentes se ha mantenido constante a partir de 2007. Sin embargo, en la generalidad de estos países la cantidad de publicaciones se ha incrementado de manera constante a partir de 2007. Un caso excepcional es Cuba, que tuvo registrada la revista Ciencias de la Información en el periodo 2000 a 2004, en los años posteriores ha perdido la acreditación. 
Tabla 1. Clasificación de las revistas según tipo de acceso e indexaciones.

\begin{tabular}{ccccc}
\hline País & Acceso libre & Acceso restringido & SciELO & WoS \\
\hline Argentina & 1 & 0 & 1 & 1 \\
Brasil & 11 & 0 & 7 & 4 \\
Chile & 3 & 0 & 3 & 1 \\
Colombia & 2 & 0 & 2 & 0 \\
México & 1 & 0 & 1 & 0 \\
Venezuela & 1 & 1 & 0 & 0 \\
Total & 19 & 1 & 14 & 6 \\
\hline
\end{tabular}

Con datos al 2019, la Tabla 1 presenta una síntesis de las 20 revistas latinoamericanas indexadas en Scopus, se observa los siguiente: el 95\% son de acceso abierto, la única revista de acceso restringido es la venezolana Espacios, que al 2021 ha sido suspendida; SciELO incluyó 14 y WoS 6; la única revista argentina presente es Mundo Agrario, está también en SciELO y WoS; Brasil tiene 11 revistas de las cuales 7 están indexadas en SciELO y 4 en WoS; las 3 revistas de Chile están en SciELO y 1 en WoS; Colombia y México tienen 2 y 1 revistas indexada en SciELO respectivamente.

En la primera columna de la Tabla 2 se presentan las 19 revistas de Latinoamérica que al 2019 son al menos Q4. Se aclara que no se consideró la Revistas brasilera de acceso abierto Atelie Geografico porque en el último año ha sido eliminada de la lista SJR.

Tabla 2. Clasificación de las revistas según tipo de acceso e indexaciones.

\begin{tabular}{|c|c|c|c|c|c|}
\hline País & Índice $\mathrm{h}$ & $\begin{array}{l}\text { Citas } 2019 \text { a artículos } \\
\text { publicados 2016-2018 }\end{array}$ & $\begin{array}{l}\text { Índice de } \\
\text { impacto JCR }\end{array}$ & $\begin{array}{l}\text { Indicador } \\
\text { SJR }\end{array}$ & $\begin{array}{l}\text { Mejor } \\
\text { cuartil }\end{array}$ \\
\hline Argentina & 8 & 18 & & & \\
\hline Mundo Agrario & 8 & 18 & 0.11 & 0.17 & Q3 \\
\hline Brasil & 75 & 552 & & & \\
\hline $\begin{array}{l}\text { BAR - Brazilian } \\
\text { Administration Review }\end{array}$ & 14 & 48 & 0.54 & 0.174 & Q4 \\
\hline Brazilian Business Review & 2 & 11 & 0.31 & 0.115 & Q4 \\
\hline Custos e Agronegocio & 6 & 92 & 0.35 & 0.206 & Q3 \\
\hline Gestao e Producao & 16 & 112 & 0.5 & 0.209 & Q3 \\
\hline $\begin{array}{l}\text { Journal of Operations and } \\
\text { Supply Chain Management }\end{array}$ & 2 & 4 & 0.27 & 0.139 & Q4 \\
\hline
\end{tabular}




\begin{tabular}{|c|c|c|c|c|c|}
\hline País & Índice $\mathrm{h}$ & $\begin{array}{l}\text { Citas } 2019 \text { a artículos } \\
\text { publicados 2016-2018 }\end{array}$ & $\begin{array}{l}\text { Índice de } \\
\text { impacto JCR }\end{array}$ & $\begin{array}{l}\text { Indicador } \\
\text { SJR }\end{array}$ & $\begin{array}{l}\text { Mejor } \\
\text { cuartil }\end{array}$ \\
\hline $\begin{array}{l}\text { RAE Revista de } \\
\text { Administracao de Empresas }\end{array}$ & 13 & 110 & 0.61 & 0.218 & Q3 \\
\hline $\begin{array}{l}\text { Revista Brasileira de Gestao } \\
\text { de Negocios }\end{array}$ & 9 & 109 & 1.23 & 0.285 & Q3 \\
\hline $\begin{array}{l}\text { Revista Brasileira de } \\
\text { Orientacao Profissional }\end{array}$ & 8 & 24 & 0.13 & 0.209 & Q4 \\
\hline $\begin{array}{l}\text { Revista Contabilidade e } \\
\text { Financas }\end{array}$ & 4 & 37 & 0.38 & 0.192 & Q4 \\
\hline $\begin{array}{l}\text { Revista de Administracao } \\
\text { Mackenzie }\end{array}$ & 1 & 5 & 0.17 & 0.104 & Q4 \\
\hline Chile & 65 & 478 & & & \\
\hline Informacion Tecnologica & 13 & 266 & 0.73 & 0.232 & Q3 \\
\hline $\begin{array}{l}\text { Journal of Technology } \\
\text { Management and Innovation }\end{array}$ & 25 & 97 & 0.57 & 0.212 & Q3 \\
\hline $\begin{array}{l}\text { Journal of Theoretical } \\
\text { and Applied Electronic } \\
\text { Commerce Research }\end{array}$ & 27 & 115 & 3.03 & 0.399 & Q2 \\
\hline Colombia & 18 & 68 & & & \\
\hline Cuadernos de administración & 8 & 14 & 0.29 & 0.141 & Q4 \\
\hline Innovar & 10 & 54 & 0.36 & 0.163 & Q4 \\
\hline México & 9 & 125 & & & \\
\hline Contaduría y administración & 9 & 125 & 0.68 & 0.174 & Q3 \\
\hline Venezuela & 24 & 1349 & & & \\
\hline Espacios (descontinuada) & 16 & 1314 & 0.34 & 0.215 & Q3 \\
\hline $\begin{array}{l}\text { Revista Venezolana de Ge- } \\
\text { rencia }\end{array}$ & 8 & 35 & 0.23 & 0.165 & Q3 \\
\hline Total & 199 & 2590 & & & \\
\hline
\end{tabular}

En la Tabla 2 se observan los nombres de revistas científica de la catagoría "negocios, administración y contabilidad” acompañadas de sus respectivos ínidices h, JCRySJR, asimismo, se especifica el mejor cuartil y la cantidad de citas recibidas en 2019 correspondiente a publicaciones del 2017 y 2018.

Los hallazgos más sobresalientes son los siguientes: 8 son Q4, 10 son Q3 y solamente la revista chilena Journal of Theoretical and Applied Electronic Commerce Research es Q2 
con un SJR 2019 de 0.399. Es decir, esta revista se encuentra dentro del $50 \%$ mejor de la categoría, además tiene el índice h más alto de la región (h27), lo que significa que tiene 27 artículos que han sido citados al menos 27 veces. Asimismo, su índice de impacto JCR 2019 es 3.03, por mucho el más alto de Latinoamérica; la revista de la misma nacionalidad Journal of Technology Management and Innovation cuenta con un índice h25; la revista Espacios con un índice h16 obtuvo 1314 citas en 2019.

La Revista Brasileira de Gestao de Negocios, tuvo en 2019 un índice JCR de 1.23, lo que significa que durante el 2019 los artículos publicados en 2017 y 2018 tuvieron en promedio 1.23 citas por artículo; la revista chilena Informacion Tecnologica tuvo un índice JCR de 0.73; RAE Revista de Administracao de Empresas alcanzó un índice JCR de 0.61.

Haciendo una comparación de los indicadores presentados en la Tabla 2, las cinco revistas con mayor impacto en Latinoamérica, presentadas de mayor a menor son: las chilenas Journal of Theoretical and Applied Electronic Commerce Research, Journal of Technology Management and Innovation e Informacion Tecnologica, las brasileras Revista Brasileira de Gestao de Negocios y RAE Revista de Administracao de Empresas. Por otra parte, considerando los mismos indicadores es posible citar las cinco revistas con menor impacto, se las enuncia a continuación de menor a mayor: Revista de Administracao Mackenzie (Brasil), Journal of Operations and Supply Chain Management (Brasil), Cuadernos de administración (Colombia), Revista Brasileira de Orientacao Profissional (Brasil) y Mundo Agrario (Argentina).

Hasta el momento se ha analizado el impacto de publicaciones científicas difundidas a través de revistas Latinoamericanas, a continuación se presenta el análisis desde la perspectiva de los países y sus investigadores.

\section{Clasificación de países (1996-2019)}

Es esta segunda etapa, el abordaje partió por observar el comportamiento en un periodo de veinticuatro años del total de países que tienen al menos un investigador que haya publicado en una revista indizada en Scopus. Nótese más adelante que desde esta perspectiva la participación latinoamericana es mayor. 


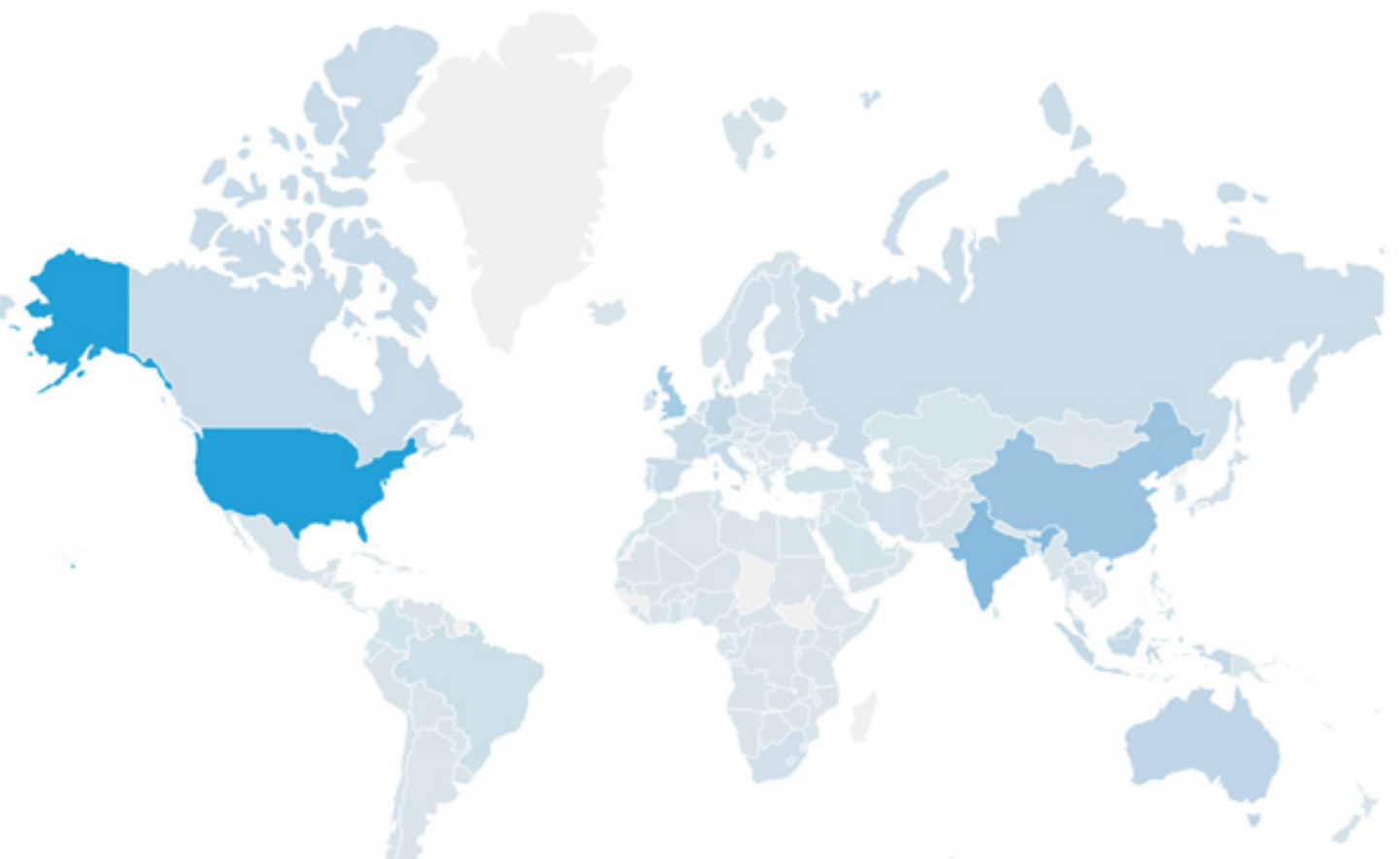

Figura 5. Mapa de publicaciones científicas por país (1996-2019) Fuente. SCImago, (2021a).

La Figura 5, presenta un mapa de resultados científicos que resalta con colores más intensos a los países que en 2019 han publicado más artículos científicos; la asignación de publicaciones se hace con base en el origen de la institución de afiliación de los autores. Resalta Estados Unidos como el país con más publicaciones del mundo, cuenta con 18371 publicaciones en 2019 y 307516 en el periodo 1996-2019; asimismo, tiene el índice h más alto 720 y la mayor cantidad de citaciones tanto en todo el periodo como en el último año estudiado. Los 10 países con mayor cantidad de publicaciones entre 1996-2019 son: Estados Unidos, Reino Unido (109417 publicaciones y h369), China (76367 publicaciones y h183), Alemania (52431 publicaciones y h243), Australia (51152 publicaciones y h265), India (45539 publicaciones y h143), Canadá (39950 publicaciones y h320), Francia (29586 publicaciones y h221), España (27819 publicaciones y h203) e Italia (26280 publicaciones y h186).

Se evidenció que, la clasificación de los países que han publicado más artículos solo en el año 2019 es, de mayor a menor: Estados Unidos, India, China, Reino Unido, Australia, Alemania, Indonesia (h59), Malaysia (h108), Italia y Canadá.

Se consideró importante resaltar que en la población estudiada no se encontró relación entre cantidad de documentos y autocitas, en el año 2019: China registró 6046 autocitas y ha publicado 10165 documentos, Estados Unidos tiene 5211 autocitas habiendo publicado 18371 documentos, Reino Unido tiene 2490 autocitas y 8236 documentos, India registró 2368 citas y 12571 documentos publicados e Italia tuvo 1273 autocitas y 35247 documentos. 
En términos de inmediatez, en el año 2019 los tres países que tuvieron una media de citaciones más alta por documento publicado el mismo año (índice de inmediatez) son: Andorra con 2 documentos publicados con una media de 17.5 citaciones por documento y un índice h5, Liechtenstein con 40 documentos, 2 citas por documento y h40, Timor-Leste un documento con 2 citas y h40.

En la Figura 6 el análisis se enfoca en la participación de los países latinoamericanos.

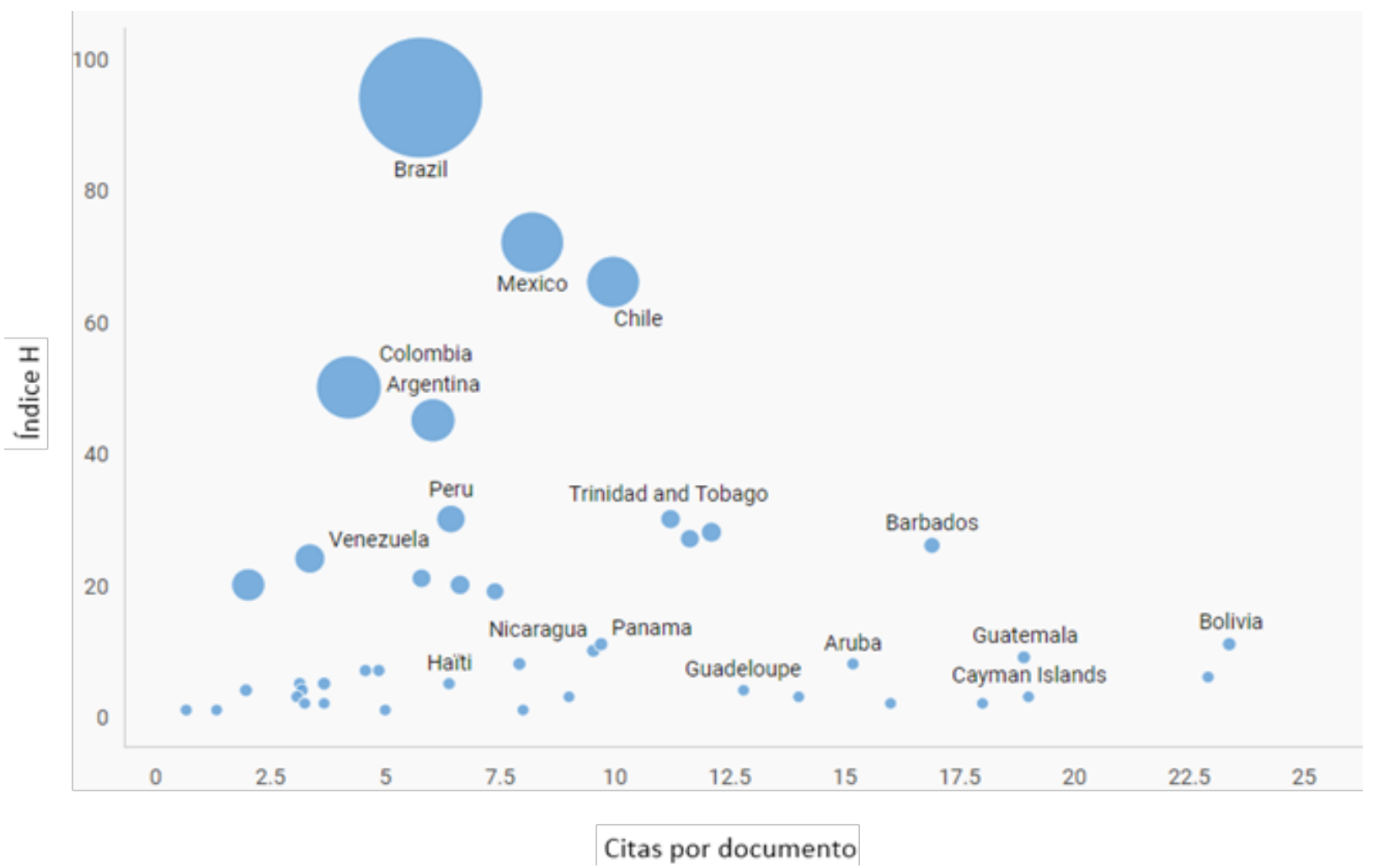

Figura 6. Cantidad de publicaciones por país (1996-2019) Fuente. SCImago, (2021b).

La Figura 6, toma los datos acumulados en el periodo 1996-2019 y correlaciona media de citas por documento con el índice $h$, tomando como objeto de estudio los países de Latinoamérica. Se observa que Brasil tiene, por mucho, un índice $\mathrm{h}$ superior al de la generalidad de los países de la región; sin embargo, su media de citas está entre las más bajas. Las estadísticas de este país son: 94h, 5.77 citas por documento y 16004 documentos publicados. Nótese que gráficamente se advierte una relación inversa entre índice h y promedio de citaciones por documento. En este caso, la media de citaciones por documento más alta es 23.37 y pertenece a Bolivia. Sin embargo, Bolivia cuenta con un índice h de 11.

En las Figuras 7 y 8 se presenta la clasificación de los 20 países de la región con mayor índice h. Asimismo, se analizan otros indicadores como documentos citables, citaciones recibidas, promedio de citaciones por documento, relación de citas, porcentaje de colaboración internacional y porcentaje de acceso abierto. 


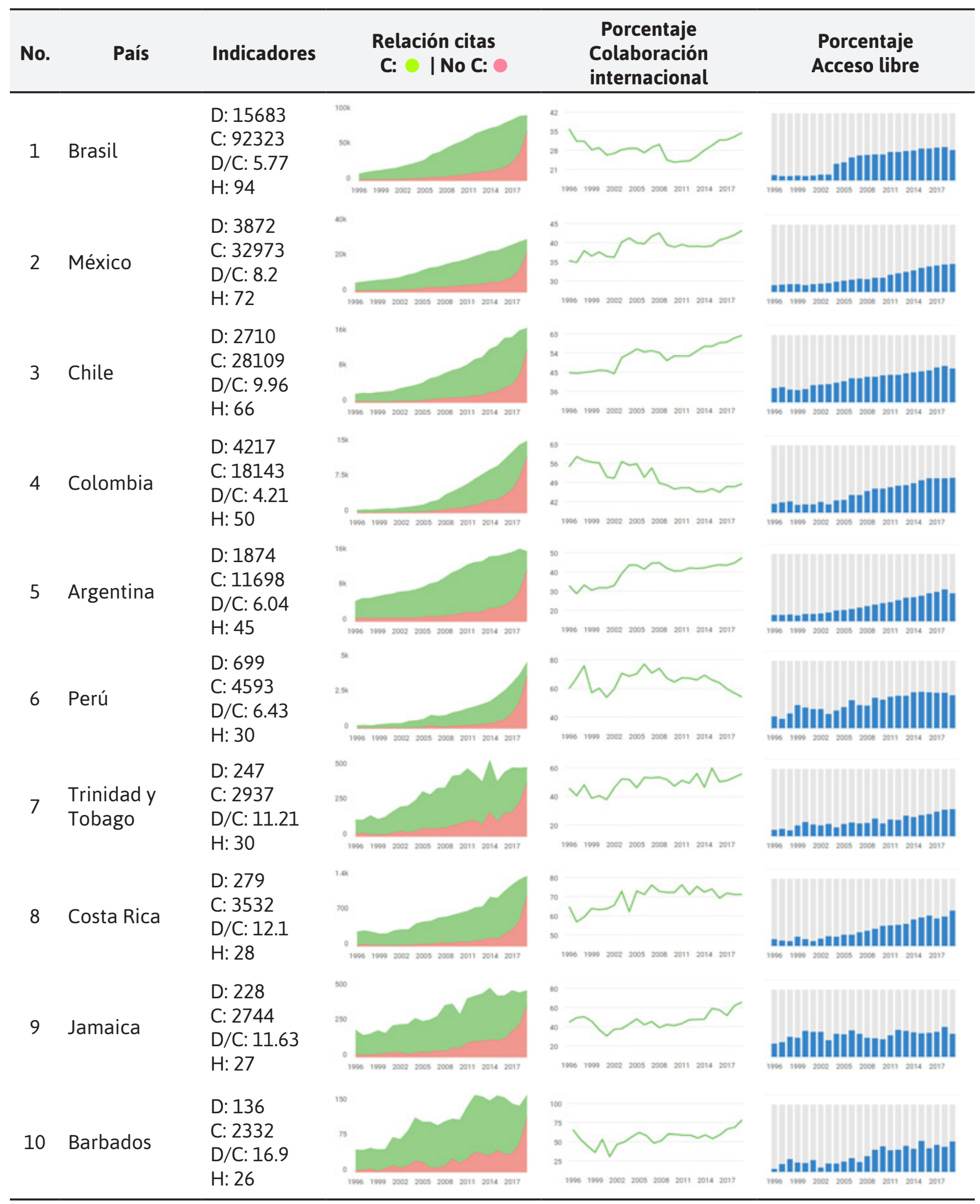

Figura 7. Clasificación de países según índice h - Lista de los diez mejores.Fuentes. SCImago (2021c); SCImago (2021d); SCImago (2021e); SCImago (2021f); SCImago (2021g); SCImago (2021h); SCImago (2021i); SCImago (2021j); SCImago (2021k); SCImago (2021l). 
Categorizando según índice h (véase clasificación en Tabla 7), las primeras 5 participaciones - Brasil, México, Chile, Colombia y Argentina - coinciden con los países con mayor cantidad de revistas científicas de Latinoamérica (Tabla 3). Sin embargo, Perú sin tener revistas científicas nacionales, con h30 (país que cuenta con 30 artículos con al menos 30 citaciones) ocupa el sexto lugar de la región. Algo similar ocurre con Trinidad y Tobago, Costa Rica, Jamaica y Barbados.

La tercera columna de la tabla 7 presenta para cada país: cantidad de documentos citables (D), citaciones recibidas (C), promedio de citaciones por documento (D/C) e índice h (H). Las gráficas presentadas en cuarta columna exponen una relación de las citas, el área verde representa el total de documento citados al menos 1 vez en los últimos 3 años y el área roja representa los documentos no citados en los últimos 3 años.

Por su parte, las gráficas presentadas en la quinta columna muestran la colaboración internacional porcentual que se entiende como la proporción de documentos cuya afiliación incluye más de una dirección de país. Resaltan los casos de Perú, Chile y Argentina en los que se advierte mayor colaboración internacional. Finalmente, la última columna presenta en color azul el porcentaje de publicaciones en bases de datos de acceso abierto y en color plomo el porcentaje de artículos publicados en bases de datos de acceso restringido.

Considerando la cantidad documentos citables para los 10 países de la Tabla 7, se tiene: Brasil aporta el 52,37\%; Colombia el 14,08\%, México el 12,93\%, Chile el 9.05\%; Argentina el 6.26\%; entre los último 5 países de la lista suman en total el restante 5.31\%, Una vez más, no existe correlación entre cantidad de revistas nacionales y publicaciones de un determinado país. En cambio, la cantidad documentos citados los resultados son los siguientes: Brasil 46.30\%; México 16.54\%; Chile 14.10\%; Colombia 9,10\%; Argentina 5.87\%, Perú 2.30\%; entre los últimos 4 países de la lista suman en total $5.79 \%$. En la Tabla 8 se analizan los puestos 11-20 de Latinoamérica, según índice h.

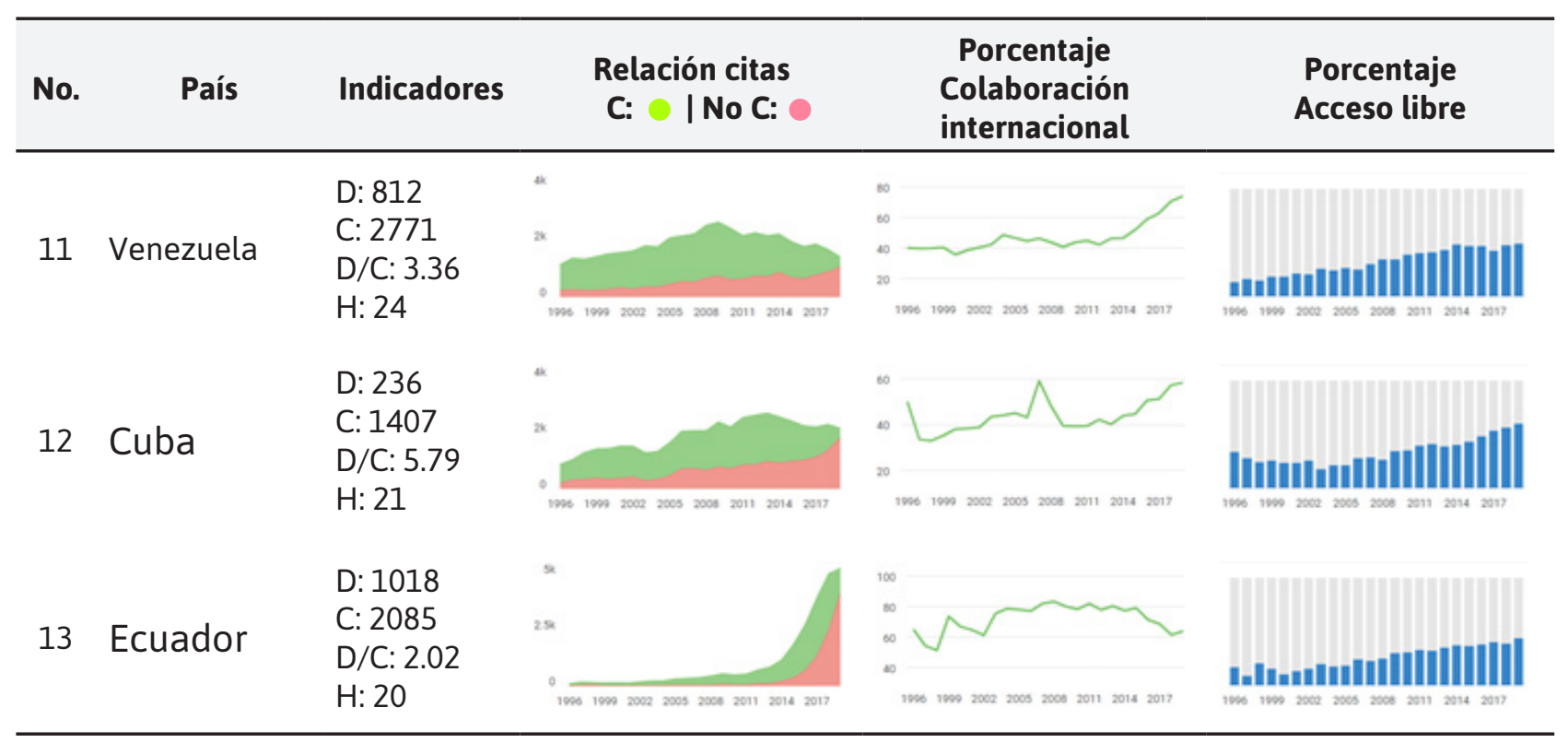




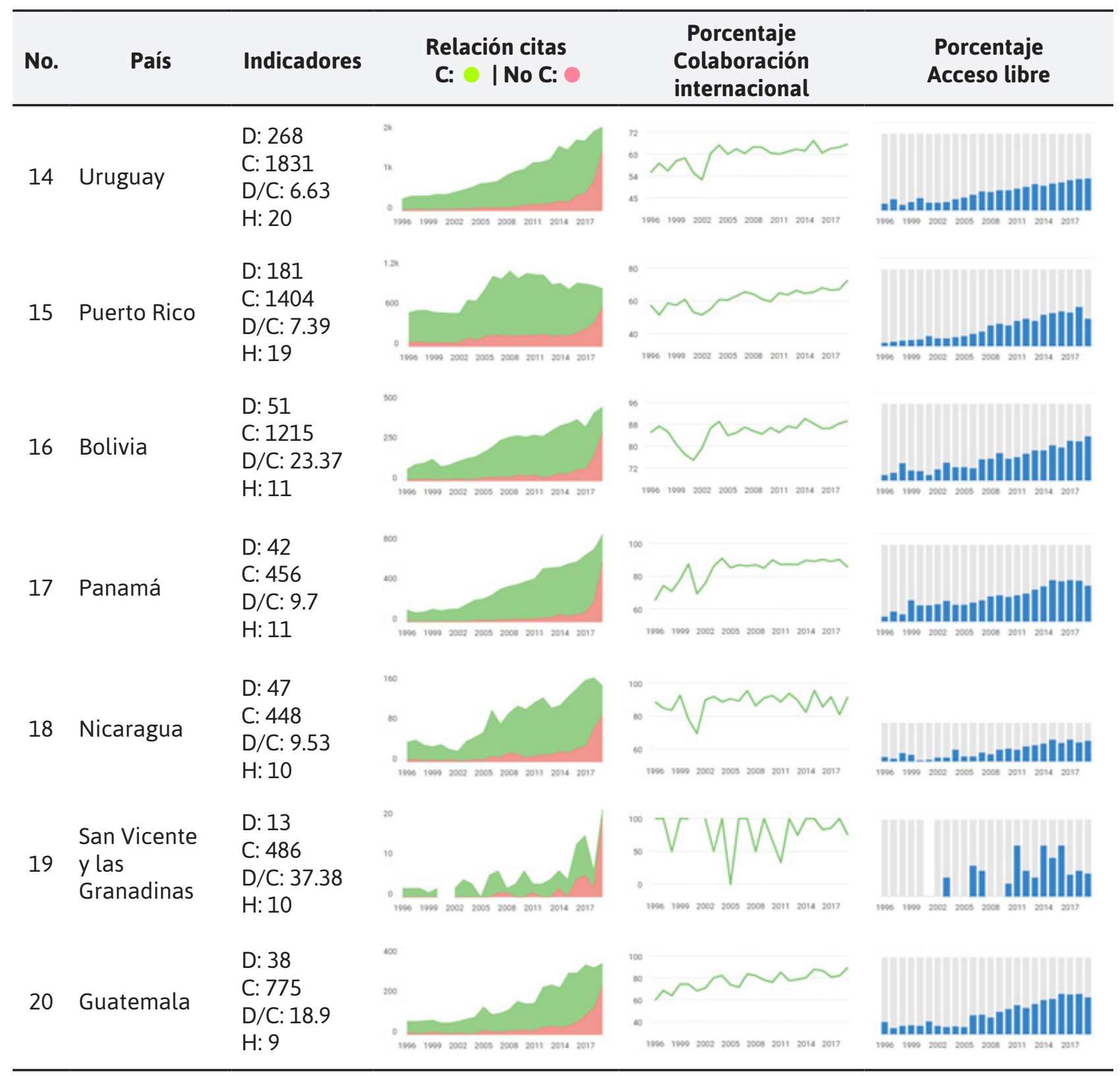

Figura 8. Clasificación de países según índice h, puestos 11-20Fuentes SCImago (2021m); SCImago (2021n); SCImago (2021o); SCImago (2021p); SCImago (2021q); SCImago (2021r); SCImago (2021s); SCImago (2021t); SCImago (2021u); SCImago (2021v).

En la Tabla 8, se siguen los criterios de la tabla 7, presentando los puestos 11-20 de la clasificación de países de Latinoamérica según índice h. Del puesto 13 al 20 figuran países que no tiene revistas indizadas a Scopus. A diferencia de la tabla anterior la media de citaciones (1288 citaciones) es representativa del grupo; sin embargo, los indicadores documentos citables, promedio de citaciones por documento e índice h, registran datos dispersos.

Según la cuarta columna de la Tabla 8, la relación de las citas (documentos citados y documentos no citados) se puntualiza: Venezuela y Cuba ha reducido significativamente su 
proporción de documentos citados a partir de 2008 y 2010 respectivamente. Observando la quinta columna, la colaboración internacional porcentual demuestra una tendencia al alza en: Venezuela, Cuba, Uruguay, Puerto Rico, Bolivia y Guatemala.

Considerando la cantidad de documentos citables para los 10 países de la Tabla 8, se tiene: Ecuador aporta el 37,62\%; Venezuela el 30,01\%; Uruguay el 9,90\%; Cuba el 8.72\%; Puerto Rico 6.69\%; Bolivia 1.88\%; Nicaragua 1.74\%; Panamá 1.55\%; Guatemala 1.40\% y San Vicente $0.48 \%$.

\section{CONCLUSIONES}

$\mathrm{M}$ ediante un estudio longitudinal de la categoría "negocios, administración y contabilidad" en el periodo 1999-2019, con un universo poblacional conformado por el total de revistas científicas indexadas en Scopus, se determinó que las revisas de mayor impacto se encuentran concentradas principalmente, entre Europa Occidental, Norte América, la Región Asiática y Europa Oriental. El impacto de Latinoamérica y Región Pacífica ha cobrado importancia a partir de 2006. A nivel Latinoamérica, a partir de 2007, resalta Brasil por el incremento de revistas científicas y Venezuela por el incremento de artículos científicos de alto impacto publicados por la revista de acceso restringido "Espacios" (revista descontinuada). Las tres únicas revistas de Chile son las de mayor impacto de la región, considerando paralelamente los índices h, JCR, SJR y la cantidad de citas recibidas. A pesar de la marcada tendencia a incrementar la cantidad de revistas y el impacto de estas, la participación de Latinoamérica es reducida. Al 2019 solo 6 países de esta región cuentan con revistas de alto impacto.

Por otra parte, considerando datos anuales de la categoría "negocios, administración y contabilidad" en el periodo 1996-2019 con un universo poblacional conformado por los países con investigaciones indexadas en Scopus, se determinó que la mayor cantidad de los autores están afiliados a instituciones de Norte América. El impacto generado por Latinoamérica y Región Pacífica ha cobrado importancia a partir de 2006. A nivel Latinoamérica, surgen estadísticas de más países, debido a que los nacionales publican en las revisas de la región que están indexadas en Scopus y en otras regiones. Brasil, México, Chile, Colombia, Argentina y Perú tienen los índices h más altos de la región.

El comportamiento histórico ha presentado fluctuaciones en el periodo analizado: si bien China ha incrementado considerablemente su participación en número de publicaciones de 1996 a 2019, su producción científica no es constante, presenta incrementos y descensos bruscos a lo largo de los años; algo similar sucede a nivel Latinoamérica en los casos se Venezuela y Cuba.

De manera general, si bien la tendencia muestra incremento en la cantidad e impacto de publicaciones en Latinoamérica, de las nueve regiones analizadas, ésta ocupa el puesto 7 según porcentaje de publicaciones citables. Por tanto, el comportamiento de las variables deberá incrementarse exponencialmente para conseguir una participación considerable.

La apropiación de los hallazgos presentados conlleva a la siguiente consideración: los dos universos poblacionales albergan solamente las revistas indexadas en Scopus. Por tanto, 
se entiende que existe una considerable cantidad de revistas indexadas en SciELO que no fueron tomadas en cuenta por no cumplir el requisito anterior. Sin embargo, esta exclusión permitió trabajar con revistas de mayor impacto mundial. Por otra parte, se entiende que existen autores de nacionalidad latina que al estar afiliados a instituciones de otras regiones no aportan a las métricas de impacto de sus países de origen. Se sugiere analizar la posibilidad de generar estadísticas que especifiquen el país de origen de los investigadores.

\section{REFERENCIAS}

Baena, J., y Alzate, I. (2020). Caracterización de las publicaciones en negocios internacionales: Estudio de caso Scimago Journal \& Country Rank. Interciencia, 45(7), 345-351

Benet, M., y Morejón, A. (2016). Posicionamiento de las revistas científicas de la salud en Cuba según el índice H5 obtenido del Google Scholar Metrics. Medisur, 14(2), 180-188

Buela, G. (2010). Índices de impacto de las revistas científicas e indicadores para medir el rendimiento de los investigadores. Revista de Psicodidáctica, 2010, 15(1), 3-19. ISSN 1136-1034

Cabezas, A. \& Torres, D. (2010). Indicadores de uso y participación en las revistas científicas 2.0: el caso de PLoS One. Profesional de la Información, 19(4), 431434. https://doi.org/10.3145/epi.2010. jul.14

Cañedo, A., y Dorta, C. (2010). SCImago Journal \& Country Rank, una plataforma para la evaluación del comportamiento de la ciencia según fuentes documentales y países. Revista Cubana de ACIMED 2010;21(3):310-320. Cuba

Delgado, P.; Ruiz, R., y Jiménez, E. (2006). Criterios MEDLINE para la selección de revistas científicas. Metodología e indicadores: Aplicación a las revistas médicas españolas con especial atención a las de salud pública. Revista Española de Salud Pública, 80(5), 521-551
Díaz, L., y Vitón, A. (2020). Análisis de la producción científica de Revista Información Científica, 2017-2019. Revista Habanera de Ciencias Médicas, 19(6), 3694

Domínguez, L.; Sixto, A.; Cogollos, L.; de Dios, J., y Benavent, A. (2018). Bibliometría e indicadores de actividad científica $(X)$. Indicadores cienciométricos en Scimago Journal and Country Rank. Análisis de la categoría temática «Pediatrics, Perinatology and Child Health». Acta Pediátrica Española, 76(7/8), E103-E108

Gómez, A.; Vargas, B.; Chinchilla, Z.; Batagelj, V., y Moya, F. (2016). Visualization and analysis of SCImago Journal \& Country Rank structure via journal clustering. Aslib Journal of information management Grupo Scimago (2008). Asia vista con el SCImago Journal \& Country Rank (SJR). Profesional de la Información, 17(6), 677678. https://doi.org/10.3145/epi.2008. nov.12

Oliván, J., y Lacruz, A. (2015). Correlación entre indicadores bibliométricos en revistas de Web of Science y Scopus. Departamento de Ciencias de la Documentación e Historia de la Ciencia. España

Ortiz, E., y Viamonte, Y. (2020). Indicadores cienciométricos y altmétricos para la identificación de líderes científicos. Palabra Clave (La Plata), 10(1), e105. https://doi.org/10.24215/18539912e105 
Romaní, F. \& Cabezas, C. (2018). Indicadores bibliométricos de las publicaciones científicas de la Revista Peruana de Medicina Experimental y Salud Pública, 2010-2017. Revista Peruana de Medicina Experimental y Salud Pública, 35, 620629

Santesteban, C. (2013). Desempeño científico argentino en cinco áreas prioritarias de I+ D: Una mirada a través del SCImago Journal \& Country Rank. CTS: Revista iberoamericana de ciencia, tecnología y sociedad, 8(22), 33-56

SCImago Research Group. (2014). Principales Indicadores Cienciométricos de la actividad científica chilena 2012, Informe 2014: Unan mirada de 10 años. Equipo de investigación conformado por: Moya-Anegón, F.; Bustos-González, A.; Chinchilla-Rodríguez, Z.; Corera-Álvarez, E.; López-Illescas, C. \& Vargas-Quesada, B. Programa Información Científica CONICYT. ISBN 123-987-6543-21-0

SCImago, (2020). SJR - SCImago Journal \& Country Rank [Portal]. 20 de october 2020. De http://www.scimagojr.com

SCImago, (2021a). SJR - SCImago Journal \& Country Rank [Portal]. 1 de enero 2021. De https://www.scimagojr.com/ countrygraph.php?area $=1400 \& y e a r=2019$

SCImago, (2021b). SJR — SCImago Journal \& Country Rank [Portal]. 1 de enero 2021. De https://www.scimagojr.com/worldreport. php?area $=1400$

SCImago, (2021c). SJR - SCImago Journal \& Country Rank [Portal]. 1 de enero 2021. De https://www.scimagojr.com/ countrysearch.php?country=BR

SCImago, (2021d). SJR - SCImago Journal \& Country Rank [Portal]. 1 de enero 2021. De https://www.scimagojr.com/ countrysearch.php?country $=M X$

SCImago, (2021e). SJR - SCImago Journal \& Country Rank [Portal]. 1 de enero 2021. De https://www.scimagojr.com/ countrysearch.php?country $=\mathrm{CL}$
SCImago, (2021f). SJR - SCImago Journal \& Country Rank [Portal]. 1 de enero 2021. De https://www.scimagojr.com/ countrysearch.php?country=CO

SCImago, (2021g). SJR - SCImago Journal \& Country Rank [Portal]. 1 de enero 2021. De https://www.scimagojr.com/ countrysearch.php?country=AR

SCImago, (2021h). SJR - SCImago Journal \& Country Rank [Portal]. 1 de enero 2021. De https://www.scimagojr.com/ countrysearch.php?country=PE

SCImago, (2021i). SJR - SCImago Journal \& Country Rank [Portal]. 1 de enero 2021. De https://www.scimagojr.com/ countrysearch.php?country=TT

SCImago, (2021j). SJR - SCImago Journal \& Country Rank [Portal]. 1 de enero 2021. De https://www.scimagojr.com/ countrysearch.php?country=CR

SCImago, (2021k). SJR - SCImago Journal \& Country Rank [Portal]. 1 de enero 2021. De https://www.scimagojr.com/ countrysearch.php?country=JM

SCImago, (2021l). SJR - SCImago Journal \& Country Rank [Portal]. 1 de enero 2021. De https://www.scimagojr.com/ countrysearch.php?country=BB

SCImago, (2021m). SJR - SCImago Journal \& Country Rank [Portal]. 1 de enero 2021. De https://www.scimagojr.com/ countrysearch.php?country=VE

SCImago, (2021n). SJR - SCImago Journal \& Country Rank [Portal]. 1 de enero 2021. De https://www.scimagojr.com/ countrysearch.php?country=CU

SCImago, (20210). SJR - SCImago Journal \& Country Rank [Portal]. 1 de enero 2021. De https://www.scimagojr.com/ countrysearch.php?country=EC

SCImago, (2021p). SJR - SCImago Journal \& Country Rank [Portal]. 1 de enero 2021. De https://www.scimagojr.com/ countrysearch.php?country=UY

SCImago, (2021q). SJR - SCImago Journal \& Country Rank [Portal]. 1 de enero 
2021. De https://www.scimagojr.com/ countrysearch.php?country=PR

SCImago, (2021r). SJR - SCImago Journal \& Country Rank [Portal]. 1 de enero 2021. De https://www.scimagojr.com/ countrysearch.php?country=BO

SCImago, (2021s). SJR - SCImago Journal \& Country Rank [Portal]. 1 de enero 2021. De https://www.scimagojr.com/ countrysearch.php?country=PA

SCImago, (2021t). SJR - SCImago Journal \& Country Rank [Portal]. 1 de enero 2021. De https://www.scimagojr.com/ countrysearch.php?country=NI

SCImago, (2021u). SJR - SCImago Journal \& Country Rank [Portal]. 1 de enero 2021. De https://www.scimagojr.com/ countrysearch.php?country=VC

SCImago, (2021v). SJR - SCImago Journal \& Country Rank [Portal]. 1 de enero 2021. De https://www.scimagojr.com/ countrysearch.php?country=GT

Spinak,E.(1998).Indicadorescienciométricos. Ciência da Informação. 27. 141-148. 10.1590/S0100-19651998000200006

Torres, D., y Jiménez, E. (2010). Introducción y estudio comparativo de los nuevos indicadores de citación sobre revistas científicas en Journal Citation Reports y Scopus. El profesional de la información, 19(2), 201-207

Urbizagastegui, R. (2016). La Bibliometría, Informetría, Cienciometría y otras "Metrías" en el Brasil. Encontros Bibli: revista eletrônica de biblioteconomia e ciência da informação, v. 21, n. 47, p. 5166, set./dez., 2016. ISSN 1518-2924. DOI: 10.5007/1518-2924.2016v21n47p51

Velásquez, T., y Tocuyo, D. (2021, february). Ubicación de revistas científicas en cuartiles según SJR: predicción a partir de estadística multivariante. In Anales de Documentación (Vol. 24, No. 1). Facultad de Comunicación y Documentación y Servicio de Publicaciones de la Universidad de Murcia. https://doi. org/10.6018/analesdoc.455951

Zacca, G., Chinchilla, Z., Vargas, B., y de Moya, F. (2014). Bibliometric analysis of regional Latin America's scientific output in public health through SCImago journal \& country rank. BMC public health, 14(1), 1-11 